\title{
Development and Validation of the Quantitative Analysis of Ampicillin Sodium in Powder for Injection by Fourier-transform Infrared Spectroscopy (FT-IR)
}

\author{
Eliane Gandolpho Tótoli, Hérida Regina Nunes Salgado*
}

Department of Drugs and Medicines, School of Pharmaceutical Sciences, UNESP - Univ Estadual Paulista, Araraquara, 14801-902, Brazil

\begin{abstract}
This paper describes the validation of an innovative analytical method for ampicillin sodium in powder for injection quantification, using Fourier-transform infrared (FT-IR) transmission spectroscopy. This technique does not use organic solvents, which is one great advantage over the most common analytical methods. This fact contributes to minimize the generation of organic solvent waste by the industry and thereby reduces the impact of its activities on the environment. The method involved absorbance measurements of the band corresponding to one of the carbonyl groups in the molecule, centered in the region between 1800 and $1700 \mathrm{~cm}^{-1}$. The method was validated according to ICH guidelines, showing to be linear $(r=0.9993)$, precise, accurate and robust, over a concentration range from 1.0 to $3.0 \mathrm{mg}$. The validated method is able to quantify ampicillin sodium in powder for injection preparation and can be used as an environmentally friendly alternative for the routine analysis in quality control.
\end{abstract}

Keywords Ampicillin Sodium, Analytical Methods, Quality Control, Spectroscopy, Quantitative, Validation

\section{Introduction}

Ampicillin is a semi-synthetic penicillin with a broad spectrum of action, which was produced in 1961 from the addition of different side chains to the penicillin nucleus[1,2]. It was the first semi-synthetic penicillin that showed activity against Gram-negative bacilli, opening the field of broad spectrum penicillins[3]. Thus, ampicillin preserves the penicillin antibacterial spectrum against Gram-positive microorganisms and it is even more active against Gram-negative, due to its increased ability to penetrate the outer membrane of these bacteria. However, it is inactivated by $\beta$-lactamases[1, 4-5]. Ampicillin sodium (AMP) (Figure 1) has the same action and use of ampicillin, considering that the sodium form is used for intramuscular and intravenous administrations[6].

There are several analytical procedures available in the literature for the analysis of ampicillin, such as high performance liquid chromatography (HPLC)[3, 7-11], fluorimetry[12], spectrophotometry[13-14] and chemilumin escence[15]. However, most of them involve the use of organic solvents, which contributes to the generation of this kind of waste by the pharmaceutical industry.

Special attention to the environmental preservation gained

* Corresponding author:

salgadoh@fcfar.unesp.br (Hérida Regina Nunes Salgado)

Published online at http://journal.sapub.org/pc

Copyright (C) 2012 Scientific \& Academic Publishing. All Rights Reserved strength since the '70s and today has a great impact on society. Thus, the trend is that the industries look for ways to reduce the impacts of their activities on the environment. In this way, they can adopt the posture of reduction, prevention or elimination of process waste. In this regard, there are some steps may be taken, including the replacement of analytical methodologies that employ organic solvents for others that do not use, the substitution of a synthesis process by another cleaner or "green" and the exchange of raw materials and supplies by other less toxic[16-17].

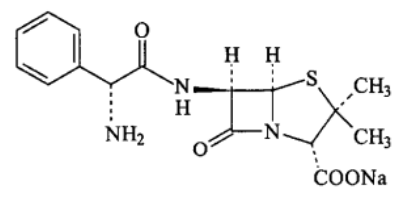

Figure 1. Chemical structure of ampicillin sodium (CAS 69-52-3)

In this context, infrared spectroscopy stands out. This is a method that does not use organic solvents and, although it is formally accepted for the identification of individual compounds, also allows the quantitation of substances. Furthermore, it is suitable for drugs with solubility problems, which can be prepared in powder form for obtaining pellets, and does not need the stage of extraction of the sample[18]. However, there are few studies in literature that use this region of the electromagnetic spectrum for this purpose[18-22].

Infrared spectroscopy is based on the fact that the molecules chemical bonds have natural vibrational 
frequencies, which correspond to its energy levels. Considering that the molecule absorbs only selected frequencies in the infrared radiation, it will only absorb those that correspond to its natural vibrational frequency, which causes an increase in the amplitude of vibrational motion of its chemical bonds. However, only the bonds that have a variation in their dipole moment over the vibration are able to absorb radiation in the infrared region. Thus, the vibration frequency can be associated with a particular bond type[23] .

The infrared spectrum of a sample can be obtained by subjecting it to a beam of infrared light. Through the examination of the transmitted light is possible to check how much energy was absorbed at each wavelength. This process can be performed using a dispersive infrared spectrometer, in which the sample is subjected to a monochromatic beam that has the frequency varied over time to obtain the data. It is also possible use a Fourier-transform spectrometer, which measures all wavelengths at once. The Fourier-transform spectrometer is the most widely used in recent times and has the advantage of being faster and more sensitive than the dispersive infrared spectrometer[23-25].

Considering all the advantages that this technique has in relation to the other methods described for the quantification of AMP in pharmaceutical formulations, the objective of this study is to propose an analytical method for AMP quantification in powder for injection preparations by FT-IR spectroscopy. Thus, this technique can be used as an environmentally friendly alternative for the routine analysis in quality control.

\section{Material and Methods}

\subsection{Chemicals}

Ampicillin sodium reference standard (AMP RS) (assigned purity 97.23\%) was kindly donated by União Química ${ }^{\circledR}$ (Brazil), as well the ampicillin sodium in powder for injection solution (Uni Ampicilin ${ }^{\circledR}$, União Química ${ }^{\circledR}$, Brazil), containing $1000 \mathrm{mg}$ of the active component. It has no excipients.

Potassium bromide $(\mathrm{KBr})$ used for the pellets preparation was analytical grade. Before use, the $\mathrm{KBr}$ was dried at $120^{\circ} \mathrm{C}$ until constant weight.

\subsection{Instrumentation and Analytical Conditions}

\subsubsection{Equipment}

The FT-IR spectrometer used was a Shimadzu ${ }^{\circledR}$ (Kyoto, Japan), model IR Prestige-21, which allows spectral digitalization for obtaining electronic files of the analysis. The region comprised in the spectral analysis was from 4000 to $500 \mathrm{~cm}^{-1}$ at $2 \mathrm{~cm}^{-1}$ intervals. After obtaining the IR spectrum and with the assistance of the IR Solution software, quantitative analysis was carried out in the spectral region between 1800 and $1700 \mathrm{~cm}^{-1}$, related to a carbonyl band of the ampicillin sodium molecule, and this band had its height analyzed in terms of absorbance.

\subsubsection{Obtaining of Analytical Curve}

Equivalent amounts of 1.0, 1.5, 2.0, 2.5 and $3.0 \mathrm{mg}$ of AMP RS (previously diluted in potassium bromide 1:10, w/w) were taken and diluted with sufficient amounts of potassium bromide to obtain $150 \mathrm{mg}$ pellets. The powders were mixed and ground until obtaining a homogeneous mixture. Thus, this mixture was compressed in a mechanical die press for 15 minutes to obtain translucent pellets, through which the beam of the spectrometer can pass.

\subsubsection{Determination of AMP in the Pharmaceutical Dosage Form}

\subsubsection{Preparation of AMP RS Pellets}

Amounts of powder equivalent to $2.0 \mathrm{mg}$ of ampicillin sodium (20.0 mg of the 1:10 dilution in potassium bromide) were taken and homogenized with $130 \mathrm{mg}$ of potassium bromide, making the total pellet weight of $150 \mathrm{mg}$. The determinations were performed in triplicate.

\subsubsection{Preparation of AMP Sample Pellets}

The contents of twenty vials of ampicillin sodium in powder for injection solution were mixed. From this mixture, amounts of $2.0 \mathrm{mg}$ of ampicillin sodium (20.0 mg of the 1:10 dilution in potassium bromide) were taken and well homogenized with $130 \mathrm{mg}$ of potassium bromide, comprising the total pellet weight of $150 \mathrm{mg}$. The determinations were performed in triplicate.

\subsubsection{Calculation of Ampicillin Sodium Content in the Sample}

The ampicillin sodium concentration in the sample was calculated by Equation 1 and its percentage content was calculated by Equation 2.

Where:

$$
\begin{aligned}
& \mathrm{Cs}=\mathrm{As} \times \frac{\mathrm{Crs}}{\mathrm{Ars}} \\
& \mathrm{Cs} \%=\mathrm{Cs} \times \frac{100}{\mathrm{Ct}}
\end{aligned}
$$

$\mathrm{Cs}=$ Sample concentration

$\mathrm{Cs} \%=$ Percentage sample concentration

$\mathrm{Crs}=$ Concentration of reference substance $(\mathrm{mg})$

As $=$ Sample absorbance

Ars $=$ Reference substance absorbance

$\mathrm{Ct}=$ Theoretical concentration of ampicillin sodium in the sample

\subsubsection{Method Validation}

The method was validated by determining the following parameters: linearity, precision, accuracy, robustness, and detection and quantification limits, according to the literature recommendation[26].

\subsubsection{Linearity}

With the intention of validate the method, five doses of AMP RS (1.0, 1.5, 2.0, 2.5 and $3.0 \mathrm{mg}$ ) were used. They were carried out in three different days. Linearity was 
evaluated by linear regression analysis, which was calculated by the least square method, and the statistical analysis was performed by ANOVA.

\subsubsection{Precision}

The precision of the method was evaluated in two requisites: repeatability and intermediate precision. Repeatability (intra-assay) was studied by the performance of seven determinations of the sample in a concentration of $2.0 \mathrm{mg} / \mathrm{pellet}$, all in the same day and identical working conditions. Intermediate precision (inter-assay) was assessed by performing the assay for a second analyst and also in three different days under the same experimental conditions. At the end of test, the relative standard deviation percentage (R.S.D.) values of the determinations were analysed[26].

\subsubsection{Accuracy}

Accuracy was attained via the recovery assay, in which known quantity of AMP RS was added to known quantity of the sample[26]. The recovery was performed in the 3 levels, $\mathrm{R} 1, \mathrm{R} 2$ and $\mathrm{R} 3$, and the pellets were prepared according to the Table 1, in triplicate.

Table 1. Preparation of pellets for the recovery assay of the method of FT-IR spectroscopy for ampicillin sodium

\begin{tabular}{ccccc} 
& $\begin{array}{c}\text { AMP } \\
\text { sample1 } \\
(\mathrm{mg})\end{array}$ & $\begin{array}{c}\text { AMP } \\
\mathrm{RS} 1 \\
(\mathrm{mg})\end{array}$ & $\begin{array}{c}\mathrm{KBr} 2 \\
(\mathrm{mg})\end{array}$ & $\begin{array}{c}\text { Final } \\
\text { concentration } \\
(\mathrm{mg} / \text { pellet })\end{array}$ \\
\hline Sample & 10,0 & - & 140,0 & 1,0 \\
R1 & 10,0 & 6,0 & 134,0 & 1,6 \\
R2 & 10,0 & 10,0 & 130,0 & 2,0 \\
R3 & 10,0 & 14,0 & 126,0 & 2,4 \\
Standard & - & 10,0 & 140,0 & 1,0
\end{tabular}

1 diluted 1:10 (w/w) in $\mathrm{KBr}$.

${ }^{2}$ sufficient amount for the preparation of pellets with a total weight of $150 \mathrm{mg}$ AMP: ampicillin sodium

AMP RS: ampicillin sodium reference standard

The recovery percentage was calculated by the equation determined by the Association of Official Analytical Chemists (AOAC)[27].

\subsubsection{Robustness}

The robustness of the method was evaluated with the purpose of showing the reliability of the analysis concerning small variations in its working parameters. In other words, it shows that the validity of the method is maintained even with small variations in its working conditions. The following parameters were individually varied: total pellet weight, brand of potassium bromide and time compression. The obtained responses were evaluated according to the R.S.D. among the dosages.

\subsubsection{Detection and Quantification Limits}

The detection (LOD) and quantification (LOQ) limits were calculated based on the intercept standard deviation and the curve slope, as described in the literature[26]. Three different curves were performed for the obtainment of the necessary data for the calculation. The values were calculated by the equations 3 and 4 .

$$
\begin{aligned}
& \mathrm{LOD}=3.3\left(\frac{\mathrm{SD}}{\mathrm{a}}\right) \\
& \mathrm{LOQ}=10\left(\frac{\mathrm{SD}}{\mathrm{a}}\right)
\end{aligned}
$$

Where:

$\mathrm{a}=$ inclination of the analytical curve

$\mathrm{SD}=$ intercept standard deviation

\section{Results and Discussion}

Quality Control is a very important step in the process of drug manufacturing, as it ensures its safety and efficacy. Thus, research on quality control of pharmaceutical products to identify the content of active and the study of physical and chemical characteristics of the drug are essential to ensure the quality of the final product.

In order to reduce environmental impacts of their activities on the environment, industries must seek alternatives to reduce, prevent or eliminate chemical residues in their routine processes. Thus, the replacement of analytical methods that employ organic solvents for others that do not employ them is an action may be taken for this purpose[16]. In that context, the infrared spectroscopy is a good option because it is a method that does not use organic solvents and also allows the quantification of compounds.

Infrared spectroscopy is based on the fact that when molecules absorb energy, undergo a transition to a state of higher energy or excited state, and only vibrational energy transitions occur in the mid-infrared region. The vibrations induced by infrared radiation include strains and tensions of inter-atomic bonds and changes of bonds angles[28]. Considering that the molecule absorbs only selected frequencies in the infrared radiation, it will only absorb those that correspond to its natural vibrational frequency, which causes an increase in the amplitude of vibrational motion of its chemical bonds. Thus, the vibration frequency can be associated with a particular bond type[23].

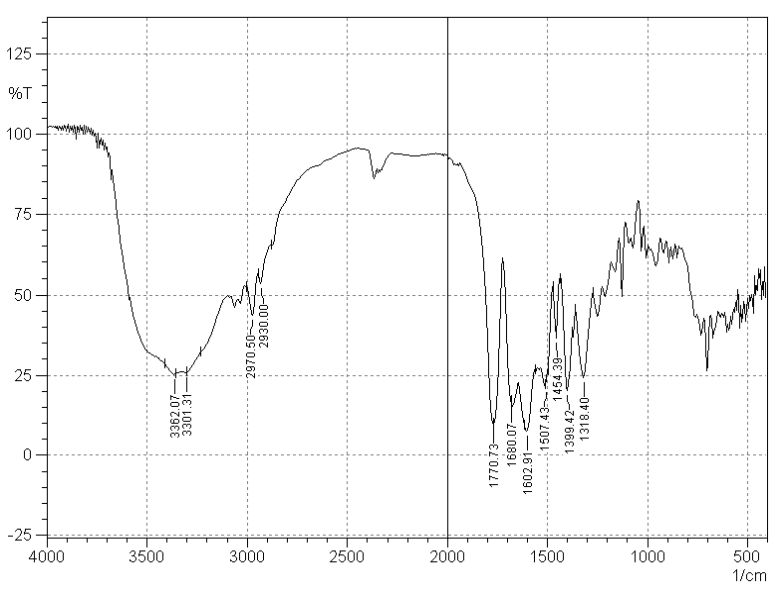

Figure 2. Infrared spectrum of AMP RS (concentration of $2 \mathrm{mg} /$ pellet) 
Besides the fact that it does not use organic solvents, the infrared spectroscopy technique has other advantages. This is a rapid technique which requires minimum or no pretreatment of the sample, provides accuracy comparable to other methods and also helps in the detection of counterfeiting and impurities. Through this technique, the sample can be scanned, on average, up to 64 times in any physical state and taking less than a minute with high resolution and precision[19]. Furthermore, it is a suitable technique for drugs with solubility problems, since they can be prepared in the pellet form[18].

The infrared spectrum of AMP RS is shown in Figure 2.

\subsection{Method Validation}

\subsubsection{Linearity}

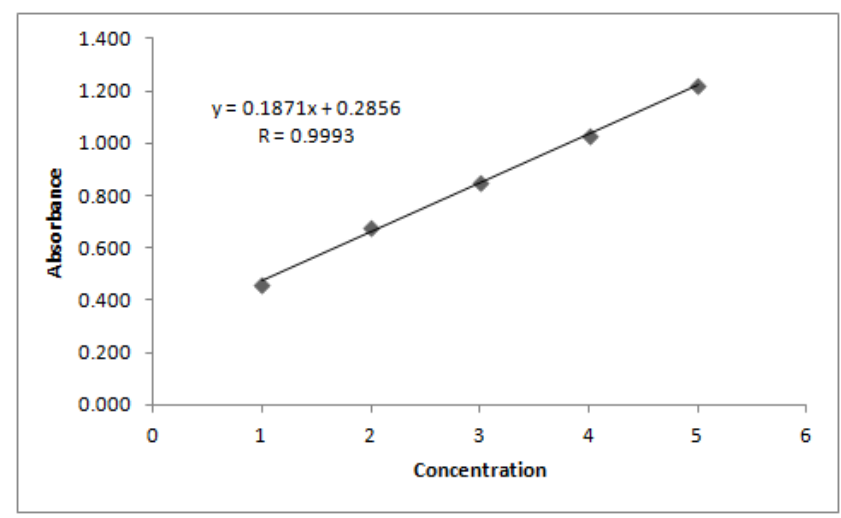

Figure 3. Graphical representation of the ampicillin sodium analytical curve by FT-IR spectroscopy

The calibration curve for ampicillin sodium RS was constructed by the disposition of the average value of absorbances in relation to their respective concentrations, showing a good linearity in the range from 1.0 to 3.0 $\mathrm{mg} /$ pellet, as shown in Figure 3. The correlation coefficient (R) presented was 0.9993 , a value close to 1.0 , which shows the excellent linearity of the method.

The ANOVA was calculated for the analytical curve data of AMP. The data showed that, statistically $(\mathrm{P}<0.05)$, there was no linearity deviation, since the F calculated (1.17) was lower than the $\mathrm{F}$ tabulated (3.71), fact that reinforces the good linearity of the method.

\subsubsection{Precision}

The precision of the method was evaluated by three parameters: intra-day or repeatability, inter-days or intermediate precision, and between analysts. The results were expressed based on the R.S.D. The intra-assay precision provided the R.S.D. value of $1.70 \%$. The R.S.D. values presented by the inter-assay and between-analysts were lower than $2.47 \%$, and are represented in the Table 2 . The data show a good precision of the method, since the R.S.D. values are close to $2 \%$ and below $5 \%$.

\subsubsection{Accuracy}

The accuracy of the method was determined by the recovery assay, which was done in three concentration levels and the results are presented in the Table 3. The data shows that the method has the adequate accuracy, meaning that the experimental concentration values are very close to the real values, since in the range of $80 \%-120 \%$, the recovery rates were close to $100 \%$.

\subsubsection{Robustness}

The robustness was evaluated by small modifications, individually, in the following method parameters: total pellet weight, brand of potassium bromide and time compression. The results are represented in the Table 4. The R.S.D. values shown are smaller than $2 \%$, showing the robustness of the analytical method for the analysis of the AMP by FT-IR spectroscopy.

Table 2. Determination of the analytical method precision for AMP by FT-IR spectroscopy

\begin{tabular}{|c|c|c|c|c|c|c|c|c|}
\hline \multicolumn{4}{|c|}{ Inter-days } & & \multicolumn{4}{|c|}{ Between analysts } \\
\hline Sample & Day & $\begin{array}{l}\text { Content }^{1} \\
(\mathrm{~g} / \text { vial })\end{array}$ & $\begin{array}{c}\text { Content }^{1} \\
(\%)\end{array}$ & $\begin{array}{c}\text { R.S.D. }{ }^{2} \\
(\%)\end{array}$ & Analyst & $\begin{array}{c}\text { Content }^{1} \\
\text { (g/vial) }\end{array}$ & $\begin{array}{c}\text { Content }^{1} \\
(\%)\end{array}$ & $\begin{array}{c}\text { R.S.D. }^{2} \\
(\%)\end{array}$ \\
\hline \multirow{3}{*}{1} & 1 & 0.984 & 98.44 & \multirow{3}{*}{1.29} & A & 0.960 & 96.01 & \multirow{3}{*}{2.47} \\
\hline & 2 & 0.960 & 96.01 & & B & 0.927 & 92.71 & \\
\hline & 3 & 0.968 & 96.75 & & & & & \\
\hline
\end{tabular}

1 average of three determinations

2 R.S.D.: relative standard deviation

Table 3. Determination of the analytical method accuracy for the analysis of AMP by FT-IR spectroscopy

\begin{tabular}{|c|c|c|c|c|c|}
\hline & Added (mg) & Found1 (mg) & Recovery1 (\%) & $\begin{array}{c}\text { Average recovery } \\
(\%)\end{array}$ & R.S.D.2 (\%) \\
\hline R1 & 0.6 & 0.60 & 99.45 & \multirow{2}{*}{100.28} & \multirow{2}{*}{0.71} \\
\hline R2 & 1.0 & 1.01 & 100.71 & & \\
\hline R3 & 1.4 & 1.41 & 100.68 & & \\
\hline
\end{tabular}

1 average of three determinations

${ }^{2}$ R.S.D.: relative standard deviation 
Table 4. Parameters of the robustness evaluation of the analytical method for the analysis of AMP by FT-IR spectroscopy

\begin{tabular}{ccccc}
\hline Variable & Range & $\begin{array}{c}\text { AMP } \\
\text { content } \\
\text { (g/vial) }\end{array}$ & $\begin{array}{c}\text { AMP } \\
\text { content } \\
(\%)\end{array}$ & $\begin{array}{c}\text { R.S.D } \\
(\%)\end{array}$ \\
\hline Total pellet & $148 \mathrm{mg}$ & 0.984 & 98.36 & \\
weight & $150 \mathrm{mg}$ & 0.992 & 99.21 & 1.27 \\
& $152 \mathrm{mg}$ & 0.968 & 96.75 & \\
KBr brand & Synth $^{\circledR}$ & 0.984 & 98.44 & \multirow{2}{*}{1.28} \\
& Shimadzu $^{\circledR}$ & 0.967 & 96.67 & \\
Time of & 10 minutes $^{\text {compression }}$ & 0.992 & 99.21 & \multirow{2}{*}{15 minutes $^{\text {Kom }}$} \\
\hline
\end{tabular}

${ }^{1}$ R.S.D.: relative standard deviation

AMP: ampicillin sodium

\subsubsection{Detection and Quantification Limits}

The detection and quantification limits were calculated in order to determine the sensibility of the method. The calculated value for the detection limit was of $0.13 \mathrm{mg}$, and the quantification limit was of $0.4 \mathrm{mg}$. The values obtained indicate the reliability of the method to detect and quantify the AMP powder for injectable preparation.

\section{Conclusions}

The results showed that the infrared method for the ampicillin sodium in powder for injection preparation quantitation presented good linearity, precision, accuracy and robustness in the range of concentrations from 1.0 to 3.0 $\mathrm{mg} / \mathrm{pellet}$. Therefore, it is a method interchangeable with other methods already described for the same purpose and can be used in routine tests for quality control of a pharmaceutical industry. Furthermore, it has some advantages over other methods described in the literature for ampicillin sodium, and the main one is that do not use organic solvents, which contributes to the non-generation of this type of residue, contributing to minimize the environmental impact of pharmaceutical industries. In addition, this study opens the possibility of applying the FT-IR spectroscopy to the quantification of other drugs.

\section{ACKNOWLEDGEMENTS}

This work was supported by PACD-FCFAr-UNESP (Araraquara, Brazil), FAPESP (São Paulo, Brazil), FUNDUNESP (São Paulo, Brazil) and CNPq (Brasília, Brazil). E.G. Tótoli was funded by FAPESP (São Paulo, Brazil) and H.R.N. Salgado was funded by CNPq (Brasília, Brazil).

\section{REFERENCES}

[1] H.P. Rang, M.M. Dale, J.M. Ritter, R.J. Flower, Rang \& Dale: Farmacologia, $6^{\text {th }}$ ed., Rio de Janeiro, Brazil: Elsevier
2007.

[2] G.N. Rolinson, and Geddes, A.M., "The 50th anniversary of the discovery of 6-aminopenicillanic acid (6-APA)", Int. J. Antimicrob. Agents, vol. 29, pp. 3-8, 2007.

[3] A.P.P. Cione, M.J. Liberale, P.M. Silva, "Development and validation of analytical method for determination of an association of ampicillins in lyophilized powder for injection by HPLC", Quím. Nova, vol. 33, no. 1, pp. 203-207, 2010.

[4] L.L. Brunton, J.S. Lazo,; K.L. Parker, Goodman \& Gilman: as bases farmacológicas da terapêutica, $11^{\text {th }}$ ed., Rio de Janeiro, Brazil: Mc Graw Hill, 2006.

[5] B.G. Katzung, Basic and Clinical Pharmacology, $10^{\text {th }}$ ed., New York: Mc Graw Hill, 2007.

[6] Remington: The Science and Practice of Pharmacy, $21^{\text {st }}$ ed., Philadelphia: Lippincott Williams \& Wilkins, 2006.

[7] M. Rambla-Alegre, R. Martí-Centelles, J. Esteve-Romero, S. Carda-Broch, "Application of a liquid chromatographic procedure for the analysis of penicillin antibiotics in biological fluids and pharmaceutical formulations using sodium dodecyl sulphate/propanol mobile phases and direct injection", J. Chromatogr. A, vol. 1218, pp. 4972-4981, 2011.

[8] R.J.M.C.L. Silva, T.A.L. Farias, J.A.R. Filho, L. Francelino, E.J.A. Oliveira, "Validation of a High-Performance Liquid Chromatography method for determination of injectable sodium ampicillin used at public hospitals in Recife, Brasil", Lat. Am. J. Pharm., vol. 29, no. 1, pp. 72-78, 2010.

[9] M. Zhou, and C. Hu, "Determination of related substances in ampicillin sodium and sulbactam sodium for injection by RP-HPLC gradient elution method", Zhongguo Kangshengsu Zazhi, vol. 34, no. 3, pp. 158-162, 2009.

[10] T.G. Barot, K. Patidar, N. Kshartri, N. Vyas, "Development and Validation of LC method for the determination of ampicillin and dicloxacillin in pharmaceutical formulation using an experimental design", E-J. Chem., vol. 6, no. 4, pp. 955-964, 2009.

[11] Z. Tan, Y. Liu, "Simultaneous content determination of ampicillin and cloxacillin for injection by HPLC", Heilongjiang Yiyao, vol. 22, no. 4, pp. 442-443, 2009.

[12] M.R. Ganjali, F. Faridbod, A.A. Saboury, P. Norouzi, "Pico-level monitoring of ampicillin by using a novel cerium fluorescence probe", Anal. Lett., vol. 43, pp. 2193-2199, 2010.

[13] T. Kurian, and J. Kurien, "Simultaneous multicomponent spectrophotometric analysis of ampicillin and probenecid in pharmaceutical formulation by derivative spectroscopy", H.J.D.Med., v. 3, n. 2, p. 57-61, 2011.

[14] G.P.A. Raghavendra, and R.V. Suryanarayana, "Spectrophotometric method for the determination of ampicillin and amoxicillin", J.P.R., vol. 3, no. 4, pp. 869-872, 2010.

[15] M.H. Sorouraddin, M. Iranifam, A. Imani-Nabiyyi, "Study of the enhancement of a new chemiluminescence reaction and its application to determination of $\beta$-lactam antibiotics", Luminescence, vol. 24, pp. 102-107, 2009.

[16] F.R. Nolasco, G.A. Tavares, J.A. Bendassolli, "Implantação 
de programas de gerenciamento de resíduos químicos laboratoriais em universidades: análise crítica e recomendações", Eng. sanit. ambient., vol. 11, no. 2, pp. 118-124, 2006.

[17] A.M. Sanseverino, "Síntese orgânica limpa", Quím. Nova, vol. 23, no. 1, pp. 102-107, 2000.

[18] A.H. Moreno, H.R.N. Salgado, "Development and validation of the quantitative analysis of ceftazidime in powder for injection by infrared spectroscopy", Physical Chemistry, vol. 2, no. 1, pp. 6-11, 2012.

[19] F. Farouk, B.A. Moussa, H.M.E-S. Azzazy, "Fourier transform infrared spectroscopy for in-process inspection, counterfeit detection and quality control of anti-diabetic drugs", Spectroscopy, vol. 26, pp. 297-309, 2011.

[20] S.T.H. Sherazi, M. Ali, S.A. Mahesar, "Application of Fourier-transform infrared (FT-IR) transmission spectroscopy for the estimation of roxithromycin in pharmaceutical formulations", Vib. Spectrosc., vol. 55, pp. 115-118, 2011.

[21] S.R. Matkovic, G.M. Vale, M. Galle, L.E. Briand, "Desarrollo y validación del análisis cuantitativo de ibuprofeno en comprimidos por espectroscopía infrarroja",
Acta Farm. Bonaerense, vol. 23, no. 4, pp. 527-532, 2004.

[22] S. Garrigues,; M. Gallignani,; M., "La Guardia, FIA-FT-IR determination of ibuprofen in pharmaceuticals", Talanta, vol. 40, no. 1, pp. 89-93, 1993.

[23] D.L. Pavia, G.M. Lampman, G.S. Kriz, J.R. Vyvyan, Introdução à espectroscopia, $4^{\text {th }}$ ed., São Paulo, Brazil: Cengage Learning, 2010.

[24] R.M. Silverstein, F.X. Webster, D.J. Kiemle, Identificação espectrométrica de compostos orgânicos, $7^{\text {th }}$ ed., Rio de Janeiro, Brazil: LTC Livros Técnicos e Científicos, 2007.

[25] G.H. Jeffrey, J. Basset, J. Mendham, R.C. Denney, Vogel: Análise química quantitativa. $5^{\text {th }}$ ed., Rio de Janeiro, Brazil: LTC Livros Técnicos e Científicos, 1992.

[26] International Harmonised Tripartite Guideline (ICH). Validation of analytical procedures: text and methodology Q2 (R1). Commission of the European Communities. Geneva, 2005.

[27] Association of Official Analytical Chemists. Official methods of analysis, 17 $7^{\text {th }}$ ed., Gaithesburg: AOAC, 2002.

[28] Brazilian Pharmacopeia, $5^{\text {th }}$. ed., Brasília, Brazil: Anvisa, 2010. 